\title{
Quantitative determination of polarization from 4D scanning electron diffraction experiments
}

\author{
Jim Ciston ${ }^{1}$, Hao Yang ${ }^{1}$, Roberto dos Reis ${ }^{1}$, Colin Ophus ${ }^{1}$, Ian Johnson ${ }^{2}$, Brent Draney ${ }^{3}$, Peter Denes ${ }^{4}$ \\ ${ }^{1}$ National Center for Electron Microscopy, Molecular Foundry, Berkeley Lab, USA \\ 2 Engineering Division, Berkeley Lab, USA \\ ${ }^{3}$ National Energy Research Scientific Computing Center, Berkeley Lab, USA \\ ${ }^{4}$ Physical Sciences, Berkeley Lab, USA
}

A vast array of new experimental modalities have been enabled in the past several years through the development of reliable pixelated detectors synchronized to probe scanning electronics to acquire the rich information present in the central portion of the convergent beam electron diffraction (CBED) pattern as a function of probe position. These four-dimensional (or more) datasets can be readily exploited for phase contrast ptychographic imaging [1], nanoscale strain mapping [2], unit cell resolution quantitative scanning position averaged convergent beam electron diffraction (S-PACBED) [3], and more.

Utilizing these new generation of high speed detectors, the size of typical datasets can be in the hundreds of gigabytes, extending to terabyte scale in the near future. Conventional pixel-by-pixel total intensity analysis of these diffraction datasets is neither sufficiently efficient or accurate to automatically analyze the quantitative content of S-PACBED patterns in these datasets for simultaneous extraction of thickness, mistilt, and polarization in complex oxide materials. Therefore, we have applied supervised learning of convolutional neural networks (CNN) to infer polarization in $\mathrm{BaTiO}_{3}$ with a precision approaching several pm displacements from single unit-cell S-PACBED signals. This method is reasonably robust to both sample thickness variations and mistilt. While CNNs have been previously demonstrated to be effective in thickness determination [5], this extension enables direct property mapping over useful fields of view without the need to form atomic resolution images.

We will also present the initial rests and design details of a CMOS Active Pixel Sensor that consists of a $576 \times 576$ array of $10 \mu \mathrm{m}$ pixels of a design related to the original TEAM detector [4] and an outer HAADF detector with 16 concentric quadrants diodes (64 elements). Data will be transported in real time via a 400 Gbps 1 km optical link to the National Energy Research Scientific Computing Center (NERSC), which will perform the 4-dimensional reconstruction and HDF5 file writing before additional asynchronous processing and analysis. The $400 \mathrm{Gbps}$ bandwidth will initially limit the frame rate to 75 $\mathrm{kHz}$, and higher rates will be obtained via in-hardware edge-computing on FPGA devices to carry out the first stage of data processing before the data is placed on the network. Compression factors of more than 100 are expected when analog signals from the detector are converted to electron events, often referred to as electron counting. [6]

\section{References:}

[1] Nellist, P. D., McCallum, B. C. \& Rodenburg, J. M., Nature, 374, 630-632 (1995).

[2] V.B. Ozdol, et al., Applied Physics Letters, 106, p. 253107 (2015).

[3] C. Ophus, P. Ercius, M. Huijben, and J. Ciston, Applied Physics Letters, 110, p. 063102 (2017).

[4] Battaglia, M., et al., Nucl. Inst. and Methods in Phys. Res. A, 622(3), p. 669 (2010).

[5] W. Xu, J.M. LeBeau, Microscopy and Microanalysis, 23(S1), 120 (2017)

[6] Research was performed at the Molecular Foundry and the National Energy Research Scientific Computing Center, DOE Office of Science User Facilities under Contract No. DE-AC02-05CH11231. JC, RdR, and HY acknowledge additional support from the DOE Early Career Research Program. 